Research Article

\title{
Pseudodifferential Operators on Weighted Hardy Spaces
}

\author{
Yu-long Deng $\mathbb{D}^{1,2}$ and Shun-chao Long $\mathbb{D}^{1}$ \\ ${ }^{1}$ School of Mathematics and Computational Science, Xiangtan University, Xiangtan 411105, China \\ ${ }^{2}$ Institute of Computational Mathematics, School of Science, Hunan University of Science and Engineering, \\ Yongzhou 425199, China \\ Correspondence should be addressed to Yu-long Deng; yuldeng@163.com and Shun-chao Long; sclong@xtu.edu.cn
}

Received 25 December 2019; Accepted 8 January 2020; Published 11 March 2020

Academic Editor: Eva A. Gallardo Gutiérrez

Copyright (c) 2020 Yu-long Deng and Shun-chao Long. This is an open access article distributed under the Creative Commons Attribution License, which permits unrestricted use, distribution, and reproduction in any medium, provided the original work is properly cited.

\begin{abstract}
We study two sufficient conditions for the boundedness of a class of pseudodifferential operators $T$ with symbols in the Hölmander class $S_{\rho, \delta}^{m}\left(\mathbb{R}^{n}\right)$ on weighted Hardy spaces $H_{\omega}^{1}\left(\mathbb{R}^{n}\right)$, where $\omega$ belongs to Muckenhoupt class $A_{p}$. The first one is an estimate from $H_{\omega}^{1}\left(\mathbb{R}^{n}\right)$ into $L_{\omega}^{1}\left(\mathbb{R}^{n}\right)$. We get a better range of admissible $p$ and $m$. The second one is a weighted version bounded for the operators $T$ on $H_{\omega}^{1}\left(\mathbb{R}^{n}\right)$, and it is an addition to the literature.
\end{abstract}

\section{Introduction}

The purpose of this paper is to study some sufficient conditions for the boundedness of pseudodifferential operators $T$ on weighted Hardy space $H_{\omega}^{1}\left(\mathbb{R}^{n}\right)$, where the operators $T$ have symbols in the Hölmander class $S_{\rho, \delta}^{m}\left(\mathbb{R}^{n}\right)$. As in [1], for $m \in \mathbb{R}$ and $\rho, \delta \in[0,1]$, a symbol $a(x, \xi) \in S_{\rho, \delta}^{m}\left(\mathbb{R}^{n}\right)$ is a smooth function defined on $\mathbb{R}^{n} \times \mathbb{R}^{n}$ such that

$$
\left|\partial_{x}^{\alpha} \partial_{\xi}^{\beta} a(x, \xi)\right| \leq C_{\alpha, \beta}(1+|\xi|)^{m-\rho|\beta|+\delta|\alpha|}
$$

holds for all multi-indices $\alpha, \beta \in \mathbb{N}^{n}$, where $C_{\alpha, \beta}$ is independent of $x$ and $\xi$. We now assume that the symbol $a(x, \xi)$ is smooth in both the spatial variable $x$ and the frequency variable $\xi$.

Given $f \in C_{0}^{\infty}\left(\mathbb{R}^{n}\right)$, the pseudodifferential operator $T \in$ $\mathscr{L}_{\rho, \delta}^{m}$ associated with the symbol $a(x, \xi) \in S_{\rho, \delta}^{m}\left(\mathbb{R}^{n}\right)$ is given by

$$
T f(x)=\int_{\mathbb{R}^{n}} a(x, \xi) \mathrm{e}^{2 \pi i x \cdot \xi} \widehat{f}(\xi) \mathrm{d} \xi,
$$

where $\widehat{f}$ denotes the Fourier transform of $f$. Moreover, we can express $T$ by a kernel $K(x, y)$ as (see, e.g., [2])

$$
T f(x)=\int K(x, y) f(y) \mathrm{d} y .
$$

Pseudodifferential operators play an important role in the theory of partial differential equations. It is well known that the Hardy spaces $H^{p}\left(\mathbb{R}^{n}\right)$ coincide with the Lebesgue spaces $L^{p}\left(\mathbb{R}^{n}\right)$ when $p>1$. The $L^{p}$ and weighted $L^{p}$ boundedness of the operator $T \in \mathscr{L}_{\rho, \delta}^{m}$ have been extensively studied. We refer to $[1,2,3,4]$ for the $L^{p}$ bounds and $[5,6,7,8]$ for the weighted $L^{p}$ bounds.

For $p \in(0,1]$, there is an estimate from $L^{1}\left(\mathbb{R}^{n}\right)$ into weak $L^{1}\left(\mathbb{R}^{n}\right)$ for the pseudodifferential operator $T \in \mathscr{L}_{\rho, \delta}^{m}$ (cf. [5]). As known, the Hardy space $H^{p}\left(\mathbb{R}^{n}\right)$ is an advantageous substitute for $L^{p}\left(\mathbb{R}^{n}\right)$. The behavior of the pseudodifferential operator $T$ on $H^{p}\left(\mathbb{R}^{n}\right)$ has attracted a lot of interest. For example, Alvarez and Hounie [5] have found that the pseudodifferential operator $T$ with symbol in $S_{\rho, \delta}^{m}\left(\mathbb{R}^{n}\right)$ is bounded from $H^{1}\left(\mathbb{R}^{n}\right)$ into $L^{1}\left(\mathbb{R}^{n}\right)$, where $0<\rho \leq 1, \quad 0 \leq \delta<1 \quad$ and $m \leq-(n / 2)(1-\rho)+\min \{0,(n$ $(\rho-\delta) / 2)\}$. Hounie and Kapp [9] have shown that the operator $T$ with $0 \leq \delta \leq \rho<1$ and $m=-(n(1-\rho)) / 2$ is bounded from the local Hardy space $h^{1}\left(\mathbb{R}^{n}\right)$ into $L^{1}\left(\mathbb{R}^{n}\right)$. Yabuta [10] has proved the operator $T$ involving a modulus of continuity $w(t)$ is bounded from $H^{1}\left(\mathbb{R}^{n}\right)$ into $L^{1}\left(\mathbb{R}^{n}\right)$.

The bounds of the pseudodifferential operator $T$ from the weighted Hardy space $H_{\omega}^{1}\left(\mathbb{R}^{n}\right)$ into the weighted Lebesgue space $L_{\omega}^{1}\left(\mathbb{R}^{n}\right)$ have also been studied. Yabuta [11] has found that the operator $T$ is bounded from $H_{\omega}^{1}\left(\mathbb{R}^{n}\right)$ into $L_{\omega}^{1}\left(\mathbb{R}^{n}\right)$, where $T \in \mathscr{L}_{1, \delta}^{0}$ and $\omega \in A_{1}$. In view of this, it is natural to look for a wide range of operator $T$ in $\mathscr{L}_{\rho, \delta}^{m}$ to study the bounds on the weighted Hardy space $H_{\omega}^{1}\left(\mathbb{R}^{n}\right)$. 
In this paper, we establish two estimates for the pseudodifferential operator $T$ with symbols in $S_{\rho, \delta}^{m}\left(\mathbb{R}^{n}\right)$. The first one is an estimate from $H_{\omega}^{1}\left(\mathbb{R}^{n}\right)$ into $L_{\omega}^{1}\left(\mathbb{R}^{n}\right)$. We extend the result in Yabuta [11] to $\omega \in A_{p}$ with $1 \leq p<1+(\varepsilon / n)$ and the operator $T$ with $0<\rho \leq 1,0 \leq \delta<1$, and $-(n+1)<m \leq$ $-(n+1)(1-\rho)$. Our first main result is stated as follows.

Theorem 1. Let $\varepsilon=\min \{1,(1+m+n / \rho)\}, p \in[1,1+(\varepsilon / n))$, $\omega \in A_{p}$ and $T \in \mathscr{L}_{\rho, \delta}^{m}$ with $0<\rho \leq 1,0 \leq \delta<1$. If $-(n+1)<$ $m \leq-(n+1)(1-\rho)$, then $T$ is bounded from $H_{\omega}^{1}\left(\mathbb{R}^{n}\right)$ into $L_{\omega}^{1}\left(\mathbb{R}^{n}\right)$, i.e., there exists a constant $C>0$ such that

$$
\|T f\|_{L_{\omega}^{1}\left(\mathbb{R}^{n}\right)} \leq C\|f\|_{H_{\omega}^{1}\left(\mathbb{R}^{n}\right)}
$$

The second one is an estimate on weighted Hardy spaces $H_{\omega}^{1}\left(\mathbb{R}^{n}\right)$ for the pseudodifferential operator $T$. It is well known that under certain conditions of $m, \rho, \delta$, the operator $T$ is bounded on $h^{1}\left(\mathbb{R}^{n}\right)(\mathrm{cf} .[9,12])$. Alvarez and Hounie [5] have found that the pseudodifferential operator $T$ is bounded on $H^{1}\left(\mathbb{R}^{n}\right)$, where $T \in \mathscr{L}_{\rho, \delta}^{m}$ with $0<\rho \leq 1,0 \leq \delta<1$, and $m \leq$ $-\lambda+\min \{0,(n(\rho-\delta) / 2)\}$ for some $((n(1-\rho)) / 2) \leq \lambda<n / 2$. It is natural to look for a weighted version estimate on $H_{\omega}^{1}\left(\mathbb{R}^{n}\right)$. We now state our second main result.

Theorem 2. Let $\mu=((1+n+m) / \rho)-n, p \in[1,(1+\mu / n))$, $\omega \in A_{p}$, and $T \in \mathscr{L}_{\rho, \delta}^{m}$ with $0<\rho \leq 1,0 \leq \delta<1$. Assume $\rho n-$ $(n+1)<m \leq-(n+1)(1-\rho)$ and $T^{*} 1=0$. Then, $T$ is bounded on $H_{\omega}^{1}\left(\mathbb{R}^{n}\right)$; i.e., there exists a constant $C>0$ such that

$$
\|T f\|_{H_{\omega}^{1}\left(\mathbb{R}^{n}\right)} \leq C\|f\|_{H_{\omega}^{1}\left(\mathbb{R}^{n}\right)}
$$

The remainder of this paper is organized as follows. In Section 2, we present some definitions and well-known results we use later. The aim of Section 3 is to set up the estimate from $H_{\omega}^{1}\left(\mathbb{R}^{n}\right)$ into $L_{\omega}^{1}\left(\mathbb{R}^{n}\right)$ for pseudodifferential operators $T$ in $\mathscr{L}_{\rho, \delta}^{m}$. We develop a method to handle $\|T a\|_{L_{\omega}^{1}\left(2^{k+1} B / 2^{k} B\right)}$ (see Proposition 1). The aim of Section 4 is to establish the estimate on weighted Hardy spaces $H_{\omega}^{1}\left(\mathbb{R}^{n}\right)$ for pseudodifferential operators $T$ in $\mathscr{L}_{\rho, \delta}^{m}$.

Most of the notations we use are standard. $C$ denotes a constant that may change from line to line and we write $a \leq b$ as shorthand for $a \leq C b$. If $a \leqq b$ and $b \leqq a$, we mean $a \sim b$. For a measurable set $A,|A|$ denotes the Lebesgue measure of $A$ and $\chi_{A}$ the characteristic function. $B$ will always denote a ball, and $t B(t>0)$ denotes the ball $B$ dilated by $t$.

\section{Notations and Auxiliary Lemma}

In this section, we first present an auxiliary lemma about the pseudodifferential operator $T$ associated with the kernel $K(x, y)$. Let $\mathcal{S}\left(\mathbb{R}^{n}\right)$ be the class of Schwartz functions and $\mathcal{S}^{\prime}\left(\mathbb{R}^{n}\right)$ be its dual space. The space of $C^{\infty}$-function with compact support is denoted by $C_{0}^{\infty}\left(\mathbb{R}^{n}\right)$. Pseudodifferential operators are bounded from $\mathcal{S}\left(\mathbb{R}^{n}\right)$ to $\mathcal{S}\left(\mathbb{R}^{n}\right)$ and so possess distribution kernels $K(x, y) \in \mathcal{S}^{\prime}\left(\mathbb{R}^{n} \times \mathbb{R}^{n}\right)$. Then, the following formula for the kernel is useful (cf. Proposition 3.1 in [9]; see also [5]).
Lemma 1. Let $a(x, \xi) \in \mathcal{S}_{\rho, \delta}^{m}\left(\mathbb{R}^{n}\right)$ with $0<\rho \leq 1,0 \leq \delta<1$, and associate with the pseudodifferential operator $T \in \mathscr{L}_{\rho, \delta}^{m}$. Then, the distribution kernel $K(x, y)$ of $T$ is smooth away from the diagonal $\left\{(x, x): x \in \mathbb{R}^{n}\right\}$ and is given by

$$
K(x, y)=\lim _{\varepsilon \longrightarrow 0} \int_{\mathbb{R}^{n}} e^{2 \pi i(x-y) \cdot \xi} a(x, \xi) \psi(\varepsilon \xi) \mathrm{d} \xi,
$$

where $\psi \in C_{0}^{\infty}\left(\mathbb{R}^{n}\right)$ satisfies $\psi(\xi)=1$ for $|\xi| \leq 1$ and the limit is taken in $\mathcal{S}^{\prime}\left(\mathbb{R}^{n}\right)$ and independent of the choice of $\psi$. If $M \in \mathbb{N}$ and $(M+m+n)>0, K(x, y)$ satisfies the estimates

$$
\sup _{|\alpha+\beta|=M}\left|D_{x}^{\alpha} D_{y}^{\beta} K(x, y)\right| \leq C_{M} \frac{1}{|x-y|^{((M+m+n) / \rho)}}, x \neq y .
$$

Moreover, for any multi-index $\alpha, \beta \in \mathbb{N}^{n}$ and $N \in \mathbb{N}$,

$$
\sup _{|x-y| \geq 1 / 2}|x-y|^{N}\left|D_{x}^{\alpha} D_{y}^{\beta} K(x, y)\right| \leq C_{\alpha, \beta, N} .
$$

The following useful $L_{\omega}^{p}$ bound for the pseudodifferential operator $T$ is obtained by Michalowski et al. [7].

Lemma 2. Let $T \in \mathscr{L}_{\rho, \delta}^{m}, \quad 0 \leq \delta<1,0<\rho \leq 1$, and $m<-n$ $(1-\rho)$. Then, for each $p \in(1, \infty)$ and $\omega \in A_{p}$, there exists a constant $C>0$ such that

$$
\|T f\|_{L_{\omega}^{p}\left(\mathbb{R}^{n}\right)} \leq C\|f\|_{L_{\omega}^{p}\left(\mathbb{R}^{n}\right)}
$$

Remark 1. Obviously, the $L_{\omega}^{2}$ bounds of pseudodifferential operators $T$ are established automatically.

Remark 2. $m \leq-(n+1)(1-\rho)$ satisfies the condition of $m$ in Lemma 2.

The following useful $L^{2}$ bound of the pseudodifferential operator $T \in \mathscr{L}_{\rho, \delta}^{m}$ is obtained by Alvarez and Hounie [5].

Lemma 3. Let $T \in \mathscr{L}_{\rho, \delta}^{m}, \quad 0 \leq \delta<1,0<\rho \leq 1$, and $m<\min \{0,(n(\rho-\delta) / 2)\}$. Then, the operator Tis bounded on $L^{2}\left(\mathbb{R}^{n}\right)$.

Remark 3. The range of $m, p$ here is $m<-n(1-\rho) \mid(1 / p)-$ $(1 / 2) \mid+\min \{0,(n(\rho-\delta) / 2)\}$ and $1<p<\infty$, respectively.

Remark 4. Obviously, $m \leq-(n+1)(1-\rho)$ satisfies the condition of $m$ in Lemma 3.

Let $p \in[1, \infty)$. A nonnegative locally integrable function $\omega$ belongs to Muckenhoupt class $A_{p}$, if there exists a constant $C>0$, such that for all balls $B \subset \mathbb{R}^{n}$,

$$
\begin{array}{r}
\left(\frac{1}{|B|} \int_{B} \omega(x) \mathrm{d} x\right)\left(\frac{1}{|B|} \int_{B}(\omega(x))^{-(1 / p-1)} \mathrm{d} x\right)^{p-1} \leq C \text { if } 1<p<\infty \\
\frac{1}{|B|} \int_{B} \omega(x) \mathrm{d} x \leq \operatorname{Cessinf}_{x \in B} \omega(x) \text { if } p=1 .
\end{array}
$$

We denote $A_{\infty}=\cup_{p \geq 1} A_{p}$.

It is well known that $\omega \in A_{p}$ implies $\omega \in A_{q}$ for all $q>p$. Also, if $\omega \in A_{p}$, then $\omega \in A_{q}$ for some $q \in[1, p)$. We thus write $q_{\omega}=\inf \left\{p \geq 1: \omega \in A_{p}\right\}$ to denote the critical index of $\omega$. For a measurable set $E$, we denote $\omega(E)=\int_{E} \omega(x) \mathrm{d} x$. The 
following lemma provides a way to compare $|E|$ and $\omega(E)$ of a set $E$ (see [13]).

Lemma 4. Let $\omega \in A_{p}$ and $p \geq 1$. Then, there exists a constant $C>0$ such that

$$
C\left(\frac{|E|}{|B|}\right)^{p} \leq \frac{\omega(E)}{\omega(B)}
$$

for all balls $B$ and measurable subsets $E \subset B$.

Given a weight function $\omega$ on $\mathbb{R}^{n}$, we denote by $L_{\omega}^{p}\left(\mathbb{R}^{n}\right)$ the weighted Lebesgue space of all functions $f$ satisfying

$$
\|f\|_{L_{\omega}^{p}\left(\mathbb{R}^{n}\right)}=\left(\int_{\mathbb{R}^{n}}|f(x)|^{p} \omega(x) \mathrm{d} x\right)^{(1 / p)}<\infty .
$$

When $p=\infty, L_{\omega}^{\infty}\left(\mathbb{R}^{n}\right)$ is $L^{\infty}\left(\mathbb{R}^{n}\right)$. Analogous to the classical Hardy space, the weighted Hardy space $H_{\omega}^{1}\left(\mathbb{R}^{n}\right)$ can be defined in terms of maximal functions.

Definition 1. Let $\omega \in A_{\infty}$. The weighted Hardy space $H_{\omega}^{1}\left(\mathbb{R}^{n}\right)$ is defined by

$$
H_{\omega}^{1}\left(\mathbb{R}^{n}\right)=\left\{f \in \mathcal{S}^{\prime}\left(\mathbb{R}^{n}\right): \phi^{*}(f)(x)=\sup _{t>0}\left|\phi_{t} * f(x)\right| \in L_{\omega}^{1}\left(\mathbb{R}^{n}\right)\right\},
$$

where $\phi \in \mathcal{S}\left(\mathbb{R}^{n}\right)$ is a fixed function with $\int \phi \mathrm{d} x \neq 0$ and $\phi_{t}(y)=\left(1 / t^{n}\right) \phi(y / t)$ for any $t>0$. Moreover, we define $\|f\|_{H_{\omega}^{1}\left(\mathbb{R}^{n}\right)}=\left\|\phi^{*}(f)\right\|_{L_{\omega}^{1}\left(\mathbb{R}^{n}\right)}$.

Remark 5. Definition 1 is independent of the choice of $\phi$ (see [14]).

Definition 2. Let $\omega$ be a weight with the critical index $q_{\omega}$. An $(1, \infty)$-atom with respect to $\omega$ is a function $a$ satisfying

$$
\operatorname{supp}(a) \subset B \quad\|a\|_{L^{\infty}} \leq \omega(B)^{-1},
$$

and $\int a(x) x^{\alpha} \mathrm{d} x=0$ for every multi-index $\alpha$ with $|\alpha| \leq\left[n\left(q_{\omega}-1\right)\right]$.

The Hardy space $H_{\omega}^{1}\left(\mathbb{R}^{n}\right)$ is a linear space spanned by all of $(1, \infty)$-atoms with respect to $\omega$. Namely, $f \in H_{\omega}^{1}\left(\mathbb{R}^{n}\right)$ if and only if $f$ can be written as (see [13])

$$
f=\sum_{j} \lambda_{j} a_{j}
$$

in the sense of $\mathcal{S}^{\prime}$, where each $a_{j}$ is an $(1, \infty)$-atom with respect to $\omega$ and $\lambda_{j}$ satisfies

$$
\sum_{j}\left|\lambda_{j}\right|<\infty
$$

Moreover, $\|f\|_{H_{\omega}^{1}\left(\mathbb{R}^{n}\right)}=\inf \left\{\sum_{j=1}^{\infty}\left|\lambda_{j}\right|: f=\sum_{j=1}^{\infty} \lambda_{j} a_{j}\right\}$.

Definition 3. Let $T$ be a pseudodifferential operator in $\mathscr{L}_{\rho, \delta}^{m}$. We say $T^{*} 1=0$ if $\int_{\mathbb{R}^{n}} T a(x) \mathrm{d} x=0$ for all $a \in L^{\infty}\left(\mathbb{R}^{n}\right)$ with compact support and $\int_{\mathbb{R}^{n}} a(x) \mathrm{d} x=0$.

\section{The Proof of Theorem 1}

In this section, we prove that the pseudodifferential operators $T$ in $\mathscr{L}_{\rho, \delta}^{m}$ are bounded from $H_{\omega}^{1}\left(\mathbb{R}^{n}\right)$ into $L_{\omega}^{1}\left(\mathbb{R}^{n}\right)$.
Proposition 1. Let $\omega \in A_{p}, p \in[1, \infty)$ and $\varepsilon=$ min $\{1,(1+m+n / \rho)\}$. Assume pseudodifferential operator $T \in \mathscr{L}_{\rho, \delta}^{m}$ with $0<\rho \leq 1, \quad 0 \leq \delta<1$, and $-(n+1)<m \leq$ $-(n+1)(1-\rho)$. Then, there exists a constant $C>0$ such that

$$
\|T a\|_{L_{\omega}^{1}\left(2^{k+1} B \backslash 2^{k} B\right)} \leq C 2^{-k(\varepsilon+n(1-p))},
$$

holds for all $(1, \infty)$-atoms a with respect to $\omega$, where $\operatorname{supp}(a) \subset B=B\left(x_{0}, r\right)$.

Proof. Inspired by the proof of Lemma 3.2 in [15], we consider two cases about the radius $r$.

Case 1. When $r \geq 1$. For every $x \in 2^{k+1} B \backslash 2^{k} B$ and $y \in B\left(x_{0}, r\right)$, we have

$$
|x-y| \geq\left|x-x_{0}\right|-\left|y-x_{0}\right| \geq 2^{k} r-r \geq 1 .
$$

Hence, by $(8)$ and properties of $(1, \infty)$-atoms with respect to $\omega$, we have

$$
\begin{aligned}
|T a(x)| & =\left|\int_{\mathbb{R}^{n}} K(x, y) a(y) \mathrm{d} y\right| \leq \int_{B}|K(x, y)||a(y)| \mathrm{d} y \\
& \leq C \int_{B} \frac{1}{|x-y|^{n+1}}|a(y)| \mathrm{d} y \leq C \frac{1}{\left|x-x_{0}\right|^{n+1}} \int_{B}|a(y)| \mathrm{d} y \\
& \leq C 2^{-k} \frac{|B|}{\left|2^{k} B\right|} \omega(B)^{-1},
\end{aligned}
$$

for all $x \in 2^{k+1} B \backslash 2^{k} B$. Thus,

$$
\|T a\|_{L_{\omega}^{1}\left(\left(2^{k+1} B\right) / 2^{k} B\right)} \leq C 2^{-k} \frac{|B|}{\left|2^{k} B\right|} \frac{\omega\left(2^{k+1} B\right)}{\omega(B)} \leq C 2^{-k(\varepsilon+n(1-p))} .
$$

Case 2. When $0<r<1$. For every $x \in\left(\left(2^{k+1} B\right) / 2^{k} B\right)$ and $y \in B\left(x_{0}, r\right)$, by moments condition, we have

$$
|T a(x)|=\left|\int_{\mathbb{R}^{n}} K(x, y) a(y) \mathrm{d} y\right| \leq \int_{B}\left|K(x, y)-K\left(x, x_{0}\right)\right||a(y)| \mathrm{d} y .
$$
have

By the mean value theorem, $1+m+n>0$, and (7), we

$$
|T a(x)| \leq C \int_{B} \frac{\left|y-x_{0}\right|}{\left|x-x_{0}\right|^{(1+m+n) / \rho}}|a(y)| \leq C \frac{r}{\left(2^{k} r\right)^{(1+m+n) / \rho}} \frac{|B|}{\omega(B)},
$$

where we take $M=1$ and use the fact that $|x-\xi| \sim\left|x-x_{0}\right|$ if $\xi \in B\left(x_{0}, r\right)$. Let us now consider two subcases.

Subcase 1. If $\left(2^{k}-1\right) r \geq 1$, then, for any $y \in B\left(x_{0}, r\right)$ and $x \in 2^{k+1} B \backslash 2^{k} B$,

$$
|x-y| \geq\left|x-x_{0}\right|-\left|y-x_{0}\right| \geq\left(2^{k}-1\right) r \geq 1 .
$$

Similar to the case $r \geq 1$, we get 


$$
|T a(x)| \leq C \frac{|B|}{\left|2^{k} B\right|} \frac{r}{\left(2^{k} r\right)^{(1+m+n / \rho)}} \omega(B)^{-1} .
$$

Since $0<r<1$ and $2^{k} r>1$, we have

$$
\frac{r}{\left(2^{k} r\right)^{(1+m+n / \rho)}} \leq \begin{cases}2^{-k(1+m+n / \rho)}, & \frac{1+m+n}{\rho} \leq 1 ; \\ 2^{-k}, & \frac{1+m+n}{\rho} \geq 1 .\end{cases}
$$

Noting $\varepsilon=\min \{1,(1+m+n / \rho)\}$, it easy to see $|T a(x)| \leq C 2^{-\varepsilon k}\left(|B| /\left|2^{k} B\right|\right) \omega(B)^{-1}$. This implies (17).

Subcase 2. If $\left(2^{k}-1\right) r<1$. Since $m \leq-(n+1)(1-\rho)$, yields

$$
\|T a\|_{L_{\omega}^{1}\left(2^{k+1} B \backslash 2^{k} B\right)} \leq \frac{C r}{\left(2^{k} r|B|\right)^{(1+m+n / \rho)}} \frac{\omega\left(2^{k+1} B\right)}{\omega(B)} \leq C 2^{-k(\varepsilon+n(1-p))} .
$$

In view of (20) and (26), we finish the proof of Proposition 1.

Proof. The proof of Theorem 1 is motivated by the atomic decomposition for $H_{\omega}^{1}\left(\mathbb{R}^{n}\right)$. Let $f \in H_{\omega}^{1}\left(\mathbb{R}^{n}\right)$. We obtain an atomic decomposition of $f$ satisfying (15) and (16). So, to prove that the pseudodifferential operators $T$ are bounded from $H_{\omega}^{1}\left(\mathbb{R}^{n}\right)$ into $L_{\omega}^{1}\left(\mathbb{R}^{n}\right)$, it suffices to show that for each $(1, \infty)$-atom $a$ with respect to $\omega$, we have $T a_{L_{\omega}^{1}\left(\mathbb{R}^{n}\right)} \leq C$. Recall that an $(1, \infty)$-atom $a$ with respect to $\omega$ is a function satisfying

$$
\begin{aligned}
\sup p(a) & \subset B, \\
\|a\|_{L^{\infty}} & \leq \omega(B)^{-1}, \\
\int a(x) d x & =0,
\end{aligned}
$$

for some ball $B=B\left(x_{0}, r\right)$.

Now, let $a$ be such an atom and write

$$
\int|T a| \omega=\int_{2 B}|T a| \omega+\int_{(2 B)^{c}}|T a| \omega=I_{1}+I_{2} .
$$

It is easy to estimate the term $I_{1}$. Using Hölder inequality and $L_{\omega}^{2}$-boundedness for the pseudodifferential operator $T$ (see Remark 1), we get

$$
\begin{aligned}
I_{1} & \leq\left(\int_{2 B}|T a|^{2} \omega\right)^{1 / 2}\left(\int_{2 B} \omega\right)^{1 / 2} \leq C\|T a\|_{L_{\omega}^{2}\left(\mathbb{R}^{n}\right)} \omega(2 B)^{1 / 2} \\
& \leq C\|a\|_{L_{\omega}^{2}\left(\mathbb{R}^{n}\right)} \omega(2 B)^{1 / 2} \leq C \omega(B)^{-1 / 2} \omega(B)^{1 / 2} \leq C,
\end{aligned}
$$

where $C$ is independent of $a$.

For the term $I_{2}$, we write

$$
I_{2}=\int_{(2 B)^{c}}|T a| \omega \leq \sum_{k=1}^{\infty} \int_{\left(2^{k+1} B \backslash 2^{k} B\right)}|T a| \omega=\sum_{k=1}^{\infty}\|T a\|_{L_{\omega}^{1}\left(2^{k+1} B \backslash 2^{k} B\right)} .
$$

By Proposition 1, we get

$$
I_{2} \leq \sum_{k=1}^{\infty} C 2^{-k(\varepsilon+n(1-p))} \leq C,
$$

since $1 \leq p<1+(\varepsilon / n)$. Combing (29) and (31), we finish the proof of Theorem 1 .

\section{The Proof of Theorem 2}

In this section, we establish the weighted norm inequality on weighted Hardy spaces $H_{\omega}^{1}\left(\mathbb{R}^{n}\right)$ for pseudodifferential operators $T$ in $\mathscr{L}_{\rho, \delta}^{m}$.

Proof. Without loss of generality, we assume $1 \leq p<1$ $+(\mu / n)$, where $\mu=(1+m+n / \rho)-n$. Fix $\phi \in \mathcal{S}\left(\mathbb{R}^{n}\right)$ and $\int_{\mathbb{R}^{n}} \phi(x) \mathrm{d} x \neq 0$. By (15), it is sufficient to show that for each $(1, \infty)$-atom $a$ with respect to $\omega,\left\|(T a)^{*}\right\|_{L_{\omega}^{1}\left(\mathbb{R}^{n}\right)} \leq C$ with $C$ independent of $a$. In order to do this, one can suppose $\operatorname{supp}(a) \subset B=B\left(x_{0}, r\right)$ and write

$$
\begin{aligned}
\left\|(T a)^{*}\right\|_{L_{\omega}^{1}\left(\mathbb{R}^{n}\right)}= & \int_{\left|x-x_{0}\right| \leq 4 r}\left|(T a)^{*}(x)\right| \omega(x) \mathrm{d} x \\
& +\int_{\left|x-x_{0}\right|>4 r}\left|(T a)^{*}(x)\right| \omega(x) \mathrm{d} x \\
= & I_{1}+I_{2} .
\end{aligned}
$$

For the term $I_{1}$, by Hölder inequality, $L_{\omega}^{2}$-boundedness of the maximal function $(T a)^{*}, L_{\omega}^{2}$-boundedness of the pseudodifferential operator $T$, and (14), we get

$$
\begin{aligned}
I_{1} & \leq\left(\int_{\left|x-x_{0}\right| \leq 4 r}\left|(T a)^{*}(x)\right|^{2} \omega(x) \mathrm{d} x\right)^{1 / 2}\left(\int_{\left|x-x_{0}\right| \leq 4 r} \omega(x) \mathrm{d} x\right)^{1 / 2} \\
& \leq C\|T a\|_{L_{\omega}^{2}\left(\mathbb{R}^{n}\right)} \omega\left(B\left(x_{0}, 4 r\right)\right)^{1 / 2} \leq C\|a\|_{L_{\omega}^{2}\left(\mathbb{R}^{n}\right)} \omega\left(B\left(x_{0}, 4 r\right)\right)^{1 / 2} \leq C,
\end{aligned}
$$

where $C$ is independent of $a$.

To estimate $I_{2}$, we first estimate $(\mathrm{Ta})^{*}(x)$ for $\left|x-x_{0}\right|$ $>4 r$. For any $t>0$, since $T^{*} 1=0$ (see Definition 3 ), we have

$$
\begin{aligned}
\left|T a * \phi_{t}(x)\right|= & \left|\int_{\mathbb{R}^{n}} \operatorname{Ta}(y) \frac{1}{t^{n}} \phi\left(\frac{x-y}{t}\right) \mathrm{d} y\right| \\
= & \left|\int_{\mathbb{R}^{n}} \operatorname{Ta}(y) \frac{1}{t^{n}}\left(\phi\left(\frac{x-y}{t}\right)-\phi\left(\frac{x-x_{0}}{t}\right)\right) \mathrm{d} y\right| \\
\leq & \frac{1}{t^{n}} \int_{\left|y-x_{0}\right|<2 r}|\operatorname{Ta}(y)|\left|\phi\left(\frac{x-y}{t}\right)-\phi\left(\frac{x-x_{0}}{t}\right)\right| \mathrm{d} y \\
& +\frac{1}{t^{n}} \int_{2 r \leq\left|y-x_{0}\right|<\left(\left|x-x_{0}\right| / 2\right)} \cdots+\frac{1}{t^{n}} \int_{\left|y-x_{0}\right| \geq\left(\left|x-x_{0}\right| / 2\right)} \cdots \\
= & E_{1}+E_{2}+E_{3} .
\end{aligned}
$$

For the term $E_{1}$, by the mean value theorem and Hölder's inequality, we have 


$$
\begin{aligned}
E_{1} \leq & \frac{1}{t^{n+1}}\|T a\|_{L^{2}\left(\mathbb{R}^{n}\right)} \\
& \times\left(\int_{\left|y-x_{0}\right|<2 r}\left|\nabla \phi\left(\frac{x-x_{0}-\gamma\left(y-x_{0}\right)}{t}\right)\right|^{2}\left|y-x_{0}\right|^{2} \mathrm{~d} y\right)^{1 / 2} \\
& \leq C \frac{r^{n+1}}{\left|x-x_{0}\right|^{n+1} \omega(B)},
\end{aligned}
$$

where $\gamma \in(0,1)$ depends on $x, y$, and $x_{0}$, and $\nabla=\left(\left(\partial / \partial x_{1}\right), \ldots,\left(\partial / \partial x_{n}\right)\right)$. Here, we use the inequalities

$$
\frac{\left|x-x_{0}-\gamma\left(y-x_{0}\right)\right|^{n+1}}{t^{n+1}}\left|\nabla \phi\left(\frac{x-x_{0}-\gamma\left(y-x_{0}\right)}{t}\right)\right| \leq C,
$$

and $\quad\left|x-x_{0}-\gamma\left(y-x_{0}\right)\right| \geq\left|x-x_{0}\right|-\gamma\left|y-x_{0}\right| \geq\left|x-x_{0}\right|-$ $\left|y-x_{0}\right| \geq\left|x-x_{0}\right| / 2$, and $L^{2}$-boundedness of the pseudodifferential operator $T$ (see Lemma 3 ).

To estimate $E_{2}$ and $E_{3}$, we first estimate $T a(y)$ when $\left|y-x_{0}\right|>2 r$ and consider two cases about $r$.

Case 3. If $r>1$, then for every $y \in \mathbb{R}^{n} \backslash B\left(x_{0}, 2 r\right)$ and $z \in B$, we have $|y-z| \geq\left|y-x_{0}\right|-\left|z-x_{0}\right| \geq r \geq 1$. Hence, by (8), we have

$$
\begin{aligned}
|T a(y)| & =\left|\int_{\mathbb{R}^{n}} K(y, z) a(z) \mathrm{d} z\right| \leq \int_{B}|K(y, z) \| a(z)| \mathrm{d} z \\
& \leq C \int_{B|y-z|^{N+n+1}}\|a\|_{L^{\infty}} \mathrm{d} z \\
& \leq C \frac{1}{\left|y-x_{0}\right|^{n+1}}\|a\|_{L^{\infty}}|B| \\
& \leq C \frac{r^{n+1}}{\left|y-x_{0}\right|^{n+1} \omega(B)} .
\end{aligned}
$$

Case 4. In the case of $0<r<1$, we have $\int_{B} a(z) \mathrm{d} z=0$. Thus, for every $y \in \mathbb{R}^{n} \backslash B\left(x_{0}, 2 r\right)$, from $1+n+m>n \rho>0$, (7) yields

$$
\begin{aligned}
|T a(y)| & =\left|\int_{\mathbb{R}^{n}} K(y, z) a(z) \mathrm{d} z\right| \\
& \leq \int_{B}\left|K(y, z)-K\left(y, x_{0}\right)\right||a(z)| \mathrm{d} z \\
& \leq C \int_{B} \frac{\left|z-x_{0}\right|}{\left|y-x_{0}\right|^{(1+n+m / \rho)}}\|a\|_{L^{\infty}} \mathrm{d} z \\
& \leq C \frac{r}{\left|y-x_{0}\right|^{(1+n+m / \rho)}}\|a\|_{L^{\infty}}|B| \\
& \leq C \frac{r^{n+1}}{\left|y-x_{0}\right|^{(1+n+m / \rho)} \omega(B)},
\end{aligned}
$$

where we use the fact that $|y-\xi| \sim\left|y-x_{0}\right|$ if $\xi \in B\left(x_{0}, r\right)$ and $\left|y-x_{0}\right|>2 r$.

Let us now continue to estimate $E_{2}$. When $r \geq 1$, using the mean value theorem and (37), we have

$$
\begin{aligned}
E_{2}= & \frac{1}{t^{n+1}} \int_{2 r \leq\left|y-x_{0}\right|<\left|x-x_{0}\right| / 2} \int_{B} 2 r \leq\left|y-x_{0}\right|<\frac{\left|x-x_{0}\right|}{2} \\
& \times\left|\nabla \phi\left(\frac{x-x_{0}-\gamma\left(y-x_{0}\right)}{t}\right)\right|\left|y-x_{0}\right| \mathrm{d} y \\
\leq & C \frac{r^{n+1}}{\left|x-x_{0}\right|^{n+1} \omega(B)} \int_{2 r \leq\left|y-x_{0}\right|<\left|x-x_{0}\right| / 2} \frac{1}{\left|y-x_{0}\right|^{n}} \mathrm{~d} y \\
\leq & C \frac{r^{n+1}}{\left|x-x_{0}\right|^{n+1} \omega(B)} \ln \left(\frac{\left|x-x_{0}\right|}{4 r}\right) .
\end{aligned}
$$

Here, we use the fact that $\left|x-x_{0}-\gamma\left(y-x_{0}\right)\right| \sim\left|x-x_{0}\right|$ under the condition of $2 r \leq\left|y-x_{0}\right|<\left(\left|x-x_{0}\right| / 2\right)$.

Similarly, in the case of $0<r<1$, by the moments condition for $a$, the mean value condition, and (38), we get

$$
\begin{aligned}
E_{2}= & \frac{1}{t^{n+1}} \int_{2 r \leq\left|y-x_{0}\right|<\left|x-x_{0}\right| / 2}\left|\int_{B}\left(K(y, z)-k\left(y, x_{0}\right)\right) a(z) \mathrm{d} z\right| \\
& \times\left|\nabla \phi\left(\frac{x-x_{0}-\gamma\left(y-x_{0}\right)}{t}\right)\right|\left|y-x_{0}\right| \mathrm{d} y \\
\leq & C \frac{r^{n+1}}{\left|x-x_{0}\right|^{n+1} \omega(B)} \int_{2 r \leq\left|y-x_{0}\right|<\left|x-x_{0}\right| / 2} \frac{1}{\left|y-x_{0}\right|^{(1+n+m / \rho)-1}} \mathrm{~d} y \\
\leq & \begin{cases}C \frac{r^{n+1}}{\left|x-x_{0}\right|^{n+1} \omega(B)} \ln \left(\frac{\left|x-x_{0}\right|}{4 r}\right), & \frac{1+m+n}{\rho}=n+1 ; \\
C \frac{r^{n+\mu}}{\left|x-x_{0}\right|^{n+\mu} \omega(B)}, & \frac{1+m+n}{\rho}<n+1 .\end{cases}
\end{aligned}
$$

For the term $E_{3}$, we have

$$
E_{3} \leq \frac{1}{t^{n}} \int_{\left|y-x_{0}\right| \geq\left|x-x_{0}\right| / 2}|\operatorname{Ta}(y)|\left(\left|\phi\left(\frac{x-y}{t}\right)\right|+\left|\phi\left(\frac{x-x_{0}}{t}\right)\right|\right) \mathrm{d} y .
$$

Since $\left|y-x_{0}\right| \geq\left(\left|x-x_{0}\right| / 2\right)$, we have $|x-y| \geq(\mid x-$ $\left.x_{0} \mid / 2\right)$. Thus,

$$
\frac{1}{t^{n}}\left|\phi\left(\frac{x-y}{t}\right)\right| \leq \frac{C}{|x-y|^{n}} \leq \frac{C}{\left|x-x_{0}\right|^{n}} .
$$

Meanwhile,

$$
\frac{1}{t^{n}}\left|\phi\left(\frac{x-x_{0}}{t}\right)\right| \leq \frac{C}{\left|x-x_{0}\right|^{n}}
$$

So, in the case of $r \geq 1$, by (37), (42), and (43), we have 


$$
\begin{aligned}
E_{3} & \leq C \int_{\left|y-x_{0}\right| \geq\left(\left|x-x_{0}\right| / 2\right)} \frac{r^{n+1}}{\left|y-x_{0}\right|^{n+1} \omega(B)} \frac{1}{\left|x-x_{0}\right|^{n}} \mathrm{~d} y \\
& =C \frac{r^{n+1}}{\left|x-x_{0}\right|^{n} \omega(B)} \int_{\left|y-x_{0}\right| \geq\left(\left|x-x_{0}\right| 2\right)} \frac{1}{\left|y-x_{0}\right|^{n+1}} \mathrm{~d} y \\
& =C \frac{r^{n+1}}{\left|x-x_{0}\right|^{n+1} \omega(B)} .
\end{aligned}
$$

In the case of $0<r<1$, by (38), (42), and (43), we have

$$
\begin{aligned}
E_{3} & \leq C \int_{\left|y-x_{0}\right| \geq\left(\left|x-x_{0}\right| / 2\right)} \frac{r^{n+1}}{\left|y-x_{0}\right|^{(1+n+m / \rho)} \omega(B)} \frac{1}{\left|x-x_{0}\right|^{n}} \mathrm{~d} y \\
& =C \frac{r^{n+1}}{\left|x-x_{0}\right|^{n} \omega(B)} \int_{\left|y-x_{0}\right| \geq\left(\left|x-x_{0}\right| / 2\right)} \frac{1}{\left|y-x_{0}\right|^{(1+n+m / \rho)}} \mathrm{d} y \\
& =C \frac{r^{n+1}}{\left|x-x_{0}\right|^{n+\mu} \omega(B)} \\
& =C \frac{r^{n+\mu}}{\left|x-x_{0}\right|^{n+\mu} \omega(B)},
\end{aligned}
$$

since $\rho n<1+n+m \leq \rho(n+1)$.

Let $x \notin B\left(x_{0}, 4 r\right)$. In view of (35), (39), (40), (44), and (45), we shall unify these formulas. Firstly, $\rho n-(n+$ $1)<m \leq(\rho-1)(n+1)$ implies $\mu \in(0,1]$. Secondly, $x \notin B$ $\left(x_{0}, 4 r\right)$ implies $\left(r /\left|x-x_{0}\right|\right)<1$. Therefore,

$$
\frac{r^{n+1}}{\left|x-x_{0}\right|^{n+1} \omega(B)} \leq \frac{r^{n+\mu}}{\left|x-x_{0}\right|^{n+\mu} \omega(B)}
$$

holds for any $\mu \in(0,1)$. Finally, since $\ln x \leq(1 / \alpha) x^{\alpha}$ holds for $x \geq 1$ and $\alpha \in(0,1)$, we have

$$
\begin{aligned}
\frac{r^{n+1}}{\left|x-x_{0}\right|^{n+1} \omega(B)} \ln \left(\frac{\left|x-x_{0}\right|}{4 r}\right) & \leq C \frac{r^{n+1}}{\left|x-x_{0}\right|^{n+1} \omega(B)}\left(\frac{\left|x-x_{0}\right|}{r}\right)^{1-\mu} \\
& =C \frac{r^{n+\mu}}{\left|x-x_{0}\right|^{n+\mu} \omega(B)} .
\end{aligned}
$$

Using these three facts, we have

$$
\left|(T a)^{*}(x)\right| \leq C \frac{r^{n+\mu}}{\left|x-x_{0}\right|^{n+\mu} \omega(B)} .
$$

Note that $p \leq 1+(\mu / n)$. Then, in all cases, we have $n+$ $\mu>n p$ and

$$
\begin{aligned}
I_{2} & \leq C \int_{\left|x-x_{0}\right|>4 r} \frac{r^{n+\mu}}{\left|x-x_{0}\right|^{n+\mu} \omega(B)} \omega(x) \mathrm{d} x \\
& =C \frac{r^{n+\mu}}{\omega(B)} \sum_{l=0}^{\infty} \int_{2^{l+2} r \leq\left|x-x_{0}\right|<2^{l+3} r} \frac{\omega(x)}{\left|x-x_{0}\right|^{n+\mu}} \\
& \leq C \sum_{l=0}^{\infty} \frac{1}{2^{l[(n+\mu)-n p]}} \\
& \leq C .
\end{aligned}
$$

This concludes the proof of Theorem 2 .

\section{Data Availability}

The author confirm that no data were used to support this study. All References used were listed.

\section{Disclosure}

This study is a part of research work done by Yu-long Deng, a $\mathrm{PhD}$ student, under the supervision of the second author.

\section{Conflicts of Interest}

The authors declare that they have no conflicts of interest.

\section{Acknowledgments}

This study was partially supported by the School of Mathematics and Computational Science, Xiangtan University, China, and Institute of Computational Mathematics, School of Science, Hunan University of Science and Engineering, China. This study was also supported by the Scientific Research Projects of Hunan Eduction Department (18C1073). The article processing charge is shared by them.

\section{References}

[1] L. Hörmander, "Pseudo-differential operators and hypoelliptic equations," Singular Integrals, vol. 10, pp. 138-183, 1967.

[2] E. M. Stein, Harmonic Analysis: Real Variable Methods, Orthogonality and Oscillatory Integrals, Princeton University Press, Princeton, NJ, USA, 1993.

[3] A. P. Calderon and R. Vaillancourt, "A class of bounded pseudo-differential operators," Proceedings of the National Academy of Sciences, vol. 69, no. 5, pp. 1185-1187, 1972.

[4] C. Feffermn, ". $L^{p}$ bounds for pseudo-differential operators," Israel Journal of Mathematics, vol. 14, no. 4, pp. 413-417, 1973.

[5] J. Alvarez and J. Hounie, "Estimates for the kernel and continuity properties of pseudo-differential operators," Arkiv För Matematik, vol. 28, no. 1-2, pp. 1-22, 1990.

[6] S. Chanillo and A. Torchinsky, "Sharp function and weighted $L^{p}$ estimates for a class of pseudodifferential operators," Arkiv för Matematik, vol. 24, no. 1-2, pp. 1-25, 1985. 
[7] N. Michalowski, D. J. Rule, and W. Staubach, "Weighted lp boundedness of pseudodifferential operators and applications," Canadian Mathematical Bulletin, vol. 55, no. 3, pp. 555-570, 2012.

[8] N. Miller, "Weighted Sobolev spaces and pseudodifferential operators with smooth symbols," Transactions of the American Mathematical Society, vol. 269, no. 1, p. 91, 1982.

[9] J. Hounie and R. A. d. S. Kapp, "Pseudodifferential operators on local Hardy spaces," Journal of Fourier Analysis and Applications, vol. 15, no. 2, pp. 153-178, 2009.

[10] K. Yabuta, "Generalizations of calderón-zygmund operators," Studia Mathematica, vol. 82, no. 1, pp. 17-31, 1985.

[11] K. Yabuta, "A note on weighted norm inequalities for pseudodifferential operators," Bulletin of the Faculty of Science, Ibaraki University. Series A, Mathematics, vol. 18, pp. 7-12, 1986.

[12] D. Goldberg, "A local version of real Hardy spaces," Duke Mathematical Journal, vol. 46, no. 1, pp. 27-42, 1979.

[13] J. García-Cuerva and J. L. Rubio De Francia, Weighted Norm Inequalities and Related Topics, North-Holland Publishing, Amsterdam, Netherlands, 1985.

[14] J. Strömberg and A. Torchinsky, Weighted Hardy Spaces, Springer-Verlag, Berlin, Germany, 1989.

[15] H. D. Hung and L. D. Ky, "An Hardy estimate for commutators of pseudo-differential operators," Taiwanese Journal of Mathematics, vol. 19, no. 4, pp. 1097-1109, 2015. 